\title{
Cervical plexus as anatomical target for the treatment of postoperative cervical neuropathic pain
}

This article was published in the following Dove Press journal: Journal of Pain Research

\author{
Jorge M Orduña Valls ${ }^{1,2}$ \\ Eliezer Soto ${ }^{2,3}$ \\ Mireya Ferrandis Martínez' \\ Carlos Nebreda ${ }^{4}$ \\ Carlos Tornero Tornero ${ }^{1,2}$ \\ 'Department of Anesthesiology, Intensive \\ Care Medicine and Pain Management, \\ Hospital Clínico Universitario de \\ Valencia, Valencia, Spain; ${ }^{2}$ Clínica Indolor \\ Valencia, Valencia, Spain; ${ }^{3}$ Millennium Pain \\ Center, Chicago, IL, USA; ${ }^{4}$ Instituto \\ Aliaga-Millennium Pain Center, Clínica \\ Teknon, Barcelona, Spain
}

Correspondence: Jorge M Orduña Valls Department of Anesthesiology, Intensive Care Medicine and Pain Management, Hospital Clínico Universitario de Valencia, Avenida Blasco Ibañez 17, Valencia 460I0, Spain

Tel +0 II 34664422436

Email dr.orduna.dolor@gmail.com

\begin{abstract}
Objective: The incidence of chronic neuropathic pain following neck dissections is approximately $40 \%$. Standard drug therapy in these patients include pharmacologic treatments due to the neuropathic pain (gabapentinoids, tricyclic antidepressants...). In this case, standard options were limited. The addition of ultrasound guidance to invasive pain management techniques has enabled us to successfully treat pathologies in which previous treatments options had been limited. Pulsed radiofrequency (PRF) ablation permits treatment over nerve structures that, due to either their morphological or functional characteristics, could not be approached using the conventional variant.
\end{abstract}

Case report: A 45-year-old man with severe postoperative pain after undergoing partial glossectomy and functional neck dissection for squamous cell carcinoma of the tongue. The patient had been treated pharmacologically for several years with minimal results, baseline VAS of 90. After a successful superficial cervical plexus block under ultrasound guidance, he underwent PRF for a possible long-lasting effect. VAS post PRF improved in subsequent visits: VAS at 1 month was 0 ; at 3 months was 10 and at 6 months was 60 .

Conclusion: Postoperative changes to include alterations in nerve structures are a frequent source of chronic pain. The incidence of this type of pain in the cervical region is quite variable. Noninvasive treatment options are limited and oftentimes ineffective. Due to its location, superficial cervical plexus is an anatomical site with the potential risk of undergoing structural alterations (fibrosis, radiotherapy-associated retraction phenomena or neuroma formation). Interventional treatments performed under ultrasound guidance allow the dynamic application of therapies such as radiofrequency ablation. PRF could potentially cause an additive effect between neuromodulation and the hydrodissection caused by the infiltration of substances within a fibrotic area.

Keywords: cervical plexus, radiofrequency, neuropathic pain, pulsed radiofrequency

\section{Introduction}

Chronic pain in postoperative head and neck cancer patients is generally associated with tumor growth, or anatomical changes that may occur during the perioperative period or after radiation therapy. Tumor growth can cause mucous ulcers, inflammation, edema, infection, and also the entrapment of neural structures eliciting intractable chronic pain. ${ }^{1}$

Surgical resection of a tumor is associated with intractable pain of the shoulder, neck, and craniofacial areas. Correct identification of structures such as the cervical plexus and the spinal accessory nerve reduces the manifestation of chronic pain 
states. After radiotherapy, some conditions such as dysphagia, mucositis, radionecrosis, xerostomy, and fibrosis are frequent and should be considered as part of our initial evaluation. $^{2}$

This type of pain has been well documented in the literature. Its prevalence reaches up to $40 \%$ following the above-mentioned interventions. ${ }^{3}$ Chaplin et $\mathrm{al}^{1}$ showed the significance of the spinal accessory nerve as the generator of chronic shoulder pain following surgical resection for oropharyngeal cancer. The importance of the spinal accessory nerve as a generator of shoulder pain and on the restriction of the joint function has long been recognized. $^{5-15}$

However, the appearance of functional alterations and pain states after other types of surgical approaches in which the spinal accessory nerve is preserved is not infrequent. $^{1,5,12,13}$

Parwis Agha-Mir-Salim et al $^{16}$ showed the essential role played by the cervical plexus in the development of chronic pain with neuropathic characteristics after several types of radical neck dissections. The cervical plexus is essential for sensory innervation of the cervical region and the skin of the shoulder. It is formed by the anterior division of the first four cervical nerves and it is a sensory anatomical substrate for this region.

The literature displays several studies on the use of pulsed radiofrequency (PRF) for many chronic pain conditions. $^{17-22}$ This radiofrequency variant has been shown to exert a biological effect unrelated to any thermal damage, but potentially focusing on both small-diameter and $\mathrm{C}$ - and A-delta nociceptive fibers. ${ }^{23}$ Vallejo et $\mathrm{al}^{24}$ showed that application of PRF could impact the expression of regulatory genes all along the nociceptive painful pathways. Subsequently, Magistroni et $\mathrm{al}^{25}$ studied the application of PRF on the brachial plexus in patients with plexus avulsion and found they obtained significant pain relief.

\section{Case description}

This is a 45-year-old male patient with the diagnosis of squamous carcinoma of the tongue originally manifested as oral leukoplakia in the right lingual area, treated with laser vaporization for several years. A head and neck MRI revealed tumor-related lymphadenopathies. Due to the extension of his disease, the patient underwent a partial right glossectomy and functional neck dissection.

During the postoperative period, the patient experienced severe (VAS 80-90) and persistent pain in the anterior neck region. The pain was treated for several years in other pain centers with neuromodulators to include gabapentinoids, tricyclic antidepressants and opioid analgesics with minimal results. The patient continued to report severe pain during his initial visits (VAS 90), localized in the retroauricular and occipital areas, as well as the upper region of the chest and shoulder. The pain was described as severe, neuropathic in nature associated with dysesthesia, allodynia, hyperalgesia, but no hypoesthesia. He also mentioned increased pain with ipsilateral rotation of the head to the affected side.

Physical examination showed dysesthesia in the territory of the following nerves: great auricular, transverse cervical and supraclavicular nerves. The patient experienced severe allodynia over the territory of the superficial cervical and supraclavicular nerves. The examination did not disclose any areas of hypoesthesia. The patient did not present any signs of motor deficits in the upper extremities, and the reflexes were normal.

With these symptoms and physical examination findings, the patient was diagnosed with chronic postoperative pain associated with the alteration of his cervical plexus. Pharmacological therapy with amitriptyline $50 \mathrm{mg}$ each 24 hrs, pregabalin $150 \mathrm{mg}$ each $12 \mathrm{hrs}$ and tapentadol $100 \mathrm{mg}$ each $12 \mathrm{hrs}$ was kept during the interventional procedure.

In view of the patient's refractoriness to the pharmacological treatment, he was offered an ultrasound-guided cervical plexus block using local anesthesia with the purpose of confirming the diagnosis. We have informed the patient about the risks and benefits secondary to the treatment with cervical plexus block and even with a possible treatment with PRF. Institutional approval (CEA EOXI from Clinic Hospital of Santiago de Compostela) was required to publish the case details and we got the patient written informed consent for publication of the case details and any accompanying images.

For the cervical plexus block, we have applied a total amount of $5 \mathrm{~mL} 0.25 \%$ levobupivacaine applied between the superficial and the deep (prevertebral) cervical fascia over C4 and C6 levels using ultrasound guidance (the protocol to identify those levels with ultrasound guidance was identifying the C7's transverse process and since that level we have counted the different levels until arriving at C4). The goal of using such a limited volume of medication was to evaluate the ability of nerve blocking to obtain pain control and after that apply some type of radio frequency with consistent options.

At the 4-week follow-up visit, the patient reported complete resolution of his symptoms during the first 2 


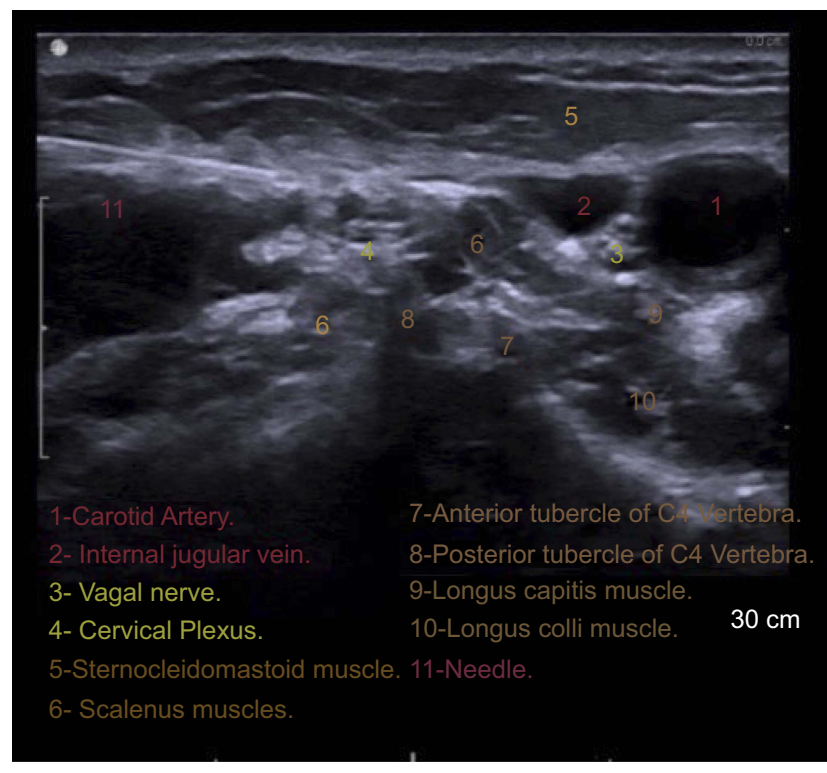

Figure I Cervical plexus at C4 level.

weeks (VAS 0). After the first 2 weeks, the pain level started to increase, reaching VAS of 20 (during the last 2 weeks). The presumed diagnosis was confirmed in view of this improvement, and a third visit was scheduled 2 weeks after. During Visit 3 (6 weeks postdiagnostic block), the patient reported recurrence of his pain to the preprocedure baseline status. Having confirmed the diagnosis using the blockade, and in view of the short duration of the patient's improvement, he was offered the application of PRF jointly with a $5-\mathrm{mL}$ injection of $0.25 \%$ levobupivacaine.

The procedure was performed in a surgical room under ultrasound guidance and local anesthesia. PRF of the cervical plexus was carried out in the interfascial compartment at $\mathrm{C} 4$ level (Figure 1). PRF protocol was the following: 8 mins of PRF on each branch, applying $45 \mathrm{~V}$, with temperature control at $42^{\circ} \mathrm{C}$, and with a pulse width of $20 \mathrm{~ms}$. After the PRF application, a total volume of $5 \mathrm{ml}$ of local anesthetic (levobupivacaine $0.2 \%$ ) was deposited at treated levels.

The patient was given a follow-up appointment on weeks 4,12 and 24 post radiofrequency application (Visits 4, 5 and 6). The test results can be seen in Table 1. With this result, we have reduced pharmacological therapy. We have discontinued tapentadol treatment, amitriptyline was reduced until $10 \mathrm{mg}$ each $24 \mathrm{hrs}$ and pregabalin was reduced until 75 each 12 hrs.

\section{Discussion}

Surgical alteration of nerve structures is a frequent source of chronic pain. The prevalence of this type of pain after
Table I Patient data

\begin{tabular}{|l|l|l|l|}
\hline Visit & VAS & DN4 & LANNS \\
\hline Baseline & 90 & 8 & 24 \\
First postdiagnostic blockade visit & 0 & 2 & 2 \\
& 20 & 3 & 5 \\
\hline Second postdiagnostic blockade visit & 90 & 8 & 24 \\
I month post-radiofrequency & 0 & 2 & 0 \\
3 months post-radiofrequency & 10 & 2 & 0 \\
6th month & 60 & 3 & 10 \\
\hline
\end{tabular}

Notes: VAS is the visual analogic scale used for graduate pain level between I and 100. The DN4 scales (which stands for Douleur Neuropathique 4) is one of the questionnaires that can be useful in diagnosing neuropathic pain. LANNS pain scale (means the Leeds assessment of neuropathic symptoms and signs) is based on analysis of sensory description and bedside examination of sensory dysfunction and provides neuropathic pain.

diverse surgeries in the cervical area is quite variable and depends upon the surgery performed. ${ }^{3}$ The potential damage of some branches (ie, spinal accessory nerve) is associated with intractable pain in the shoulder and neck area. Correct identification and avoidance of those branches during the surgery allows for better outcomes. In addition, radiotherapy treatment may cause some known conditions such as dysphagia, mucositis, radionecrosis, xerostomia and fibrosis that are associated with severe pain and discomfort. ${ }^{2}$ These conditions are usually difficult to diagnose and are frequently undertreated, being that noninvasive treatment options usually insufficient.

Parwis Agha-Mir-Salim et $\mathrm{al}^{16}$ described the essential role played by the cervical plexus in this neuropathic condition. This plexus is essential for the sensory innervation of the cervical region which makes it an important target in interventional pain treatments. Ultrasound guidance makes it possible to obtain direct and dynamic visualization of the neck-innerving structures. It also allows to visualize postsurgical changes such as neuromas, adhesions phenomena by fibrosis, and direct visualization of different therapies such as adhesion release, antiinflammatory drugs deposit, or application of different types of radiofrequency ablation.

Due to its location, the cervical plexus is an anatomical structure with many risks for procedural complications. For example, fibrosis may result due to sutures applied directly into the nerve for surgical wound closure, and/or secondary to the use of radiation therapy. Hydrodissection therapy can be an option in patients with this condition;, nevertheless, the short improvement showed with the diagnostic block makes us select the application of radiofrequency ablation as a more effective therapy. 
PRF has proven to be a neuromodulating therapy by several studies. ${ }^{23,24}$ Its application onto a damaged nervous site could revert the hypersensitivity effects developed because of the expression of regulatory genes along the nociceptive painful pathways. ${ }^{24}$ However, due to its location and histological features, radiofrequency ablation to the cervical plexus can carry several complications including cutaneous lesions, dysesthesias and vessels damages among others.

PRF enables to sum up demonstrated neuromodulator's effects with the possible hydrodissection phenomenon caused by the injection of corticosteroids and local anesthetics in a fibrotic area. Its security in this superficial location allows to treat a damaged structure. Conventional radiofrequency is also a possible treatment option in this location; however, it still has yet to be demonstrated its ability to improve outcome in this dangerous location. This case report has demonstrated that the cervical plexus is a possible target for interventional techniques for chronic postoperative pain.

\section{Acknowledgments}

Thanks goes to the Anesthesia and Pain Management Department of Hospital Clínico Universitario de Santiago de Compostela and its Chairman Dr. Alvarez for financial support as well as the Anesthesia and Pain Management Department Hospital Clínico Universitario de Valencia and its Chairman Dr. Belda for all their support.

\section{Disclosure}

The authors report no conflicts of interest in this work.

\section{References}

1. Chaplin JM, Morton RP. A prospective, longitudinal study of pain in head and neck cancer patients. Head Neck. 1999;21(6):531-537.

2. Vecht CJ, Hoff AM, Kansen PJ, de Boer MF, Bosch DA. Types and causes of pain in cancer of the head and neck. Cancer. 1992;70(1):178-184.

3. Keefe FJ, Gerdenio MSJ, Brantley A, Crisson J. Pain in the head and neck cancer patient: changes over treatment. Head Neck Surg. 1986;8:169-176. doi:10.1002/hed.2890080308

4. Krause HR. Shoulder-arm-syndrome after radical neck dissection: its relation with the innervation of the trapezius muscle. Int $J$ Oral Maxillofac Surg. 1992;21(5):276-279.

5. Short SO, Kaplan JN, Laramore GE, Cummings CW. Shoulder pain and function after neck dissection with or without preservation of the spinal accessory nerve. Am J Surg. 1984;148(4):478-482.

6. Saunders JR Jr, Hirata RM, Jaques DA. Considering the spinal accessory nerve in head and neck surgery. Am J Surg. 1985;150(4):491-494.

7. Krause HR, Bremerich A, Hermann M. The innervation of the trapezius muscle in connection with radical neck dissection: an anatomical study. J Craniomaxillofac Surg. 1991;19(2):87-89.
8. Remmler D, Byers R, Scheetz J, Shell B, White G, Zimmerman S. A prospective study of shoulder disability resulting from radical and modified neck dissections. Head Neck Surg. 1986;8(4):280-286.

9. Saunders WH, Johnson EW. Rehabilitation of the shoulder after radical neck dissection. Ann Otol Rhinol Laryngol. 1975;84 (6):812-816. doi:10.1177/000348947508400613

10. Cantlon GE, Gluckman JL. Sternoclavicular joint hypertrophy following radical neck dissection. Head Neck Surg. 1983;5(3):218-221.

11. Pfeifle K, Koch H. Pain syndromes as late sequelae of neck dissection. Schmerzsyndrome als Spatfolge nach "Neck dissection". Dtsch Zahnarztl Z. 1973;28(10):968-972.

12. Patten C, Hillel AD. The 11th nerve syndrome: accessory nerve palsy or adhesive capsulitis? Arch Otolaryngol Head Neck Surg. 1993;119 (2):215-220.

13. Bocca E, Pignataro O, Sasaki CT. Functional neck dissection: a description of operative technique. Arch Otolaryngol. 1980;106 (9):524-527.

14. Robbins KT, Medina JE, Wolfe GT, Levine PA, Sessions RB, Pruet CW. Standardizing neck dissection terminology: official report of the Academy's Committee for Head and Neck Surgery and Oncology. Arch Otolaryngol Head Neck Surg. 1991;117 (6):601-605.

15. Parwis Agha-Mir-Salim W, Schulte-Mattler U, Funk C, Lautenschläger M, Bloching M. Berghaus A. Origin of shoulder pain after "neck dissection". Importance of the cervical plexus. HNO. 2002;50(6):544-552.

16. Van Kleef M, Barendse GAM, Dingemans WAA, Wingen C, Lousberg R, de Lange S. Effects of producing a radiofrequency lesion adjacent to the dorsal root ganglion in patients with thoracic segmental pain. Clin J Pain. 1995;11(4):325-332.

17. Martin DC, Willis ML, Mullinax LA, Clarke NL, Homburger JA, Berger IH. Pulsed radiofrequency application in the treatment of chronic pain. Pain Pract. 2007;7(1):31-35. doi:10.1111/j.15332500.2007.00107.x

18. Van Zundert J, Patijn J, Kessels A, Lam'E I, van Suijlekom H, Van Kleef M. Pulsed radiofrequency adjacent to the cervical dorsal root ganglion in chronic cervical radicular pain: a double blind sham-controlled randomized clinical trial. Pain. 2007;127(1-2):173-182. doi:10.1016/j.pain.2006.09.002

19. Gallagher RM. Pulsed radiofrequency treatment: what is the evidence of its effectiveness and should it be used in clinical practice? Pain Med. 2006;7(5):408-410. doi:10.1111/j.15264637.2006.00211.x

20. Van Zundert J, De Louw AJA, Joosten EAJ, Kessels AG, Honig W, Dederen PJ. Pulsed and continous radiofrequency current adjacent to the cervical dorsal root ganglion of the rat induces late cellular activity in the dorsal horn. Anesthesiology. 2005;102(1):125-131.

21. Podhajsky RJ, Sekiguchi Y, Kikuchi S, Myers RR. The histologic effects of pulsed and continuous radiofrequency lesions at 42 degrees $\mathrm{C}$ to rat dorsal root ganglion and sciatic nerve. Spine. 2005;30 (9):1008-1013.

22. Hamann W, Abou-Sherif S, Thompson S, Hall S. Pulsed radiofrequency applied to dorsal root ganglia causes a selective increase in ATF3 in small neurons. Eur $J$ Pain. 2006;10(2):171-176. doi:10.1016/j.ejpain.2005.03.001

23. Vallejo R, Tilley DM, Williams J, Labak S, Aliaga L, Benyamin RM. Pulsed radiofrequency modulates pain regulatory gene expression along the nociceptive pathway. Pain Physician. 2013;16(5):E601-E613.

24. Magistroni E, Ciclamini D, Panero B, Verna V. Ultrasound-guided pulse-dose radiofrequency: treatment of neuropathic pain after brachial plexus lesion and arm revascularization. Case Rep Med. 2014;2014:429618. doi:10.1155/2014/429618 


\section{Publish your work in this journal}

The Journal of Pain Research is an international, peer reviewed, open access, online journal that welcomes laboratory and clinical findings in the fields of pain research and the prevention and management of pain.

Original research, reviews, symposium reports, hypothesis formation

management system is completely online and includes a very quick and fair peer-review system, which is all easy to use. Visit http:// www.dovepress.com/testimonials.php to read real quotes from puband commentaries are all considered for publication. The manuscript

lished authors. 